\title{
Una propuesta didáctica para el aprendizaje de la lengua francesa a través del «slam».
}

\author{
Brigitte Urbano Marchi \\ Departamento de Didáctica de la Lengua y la Literatura \\ Universidad de Granada
}

Recibido: 6 marzo 2008 / Aceptado: 9 mayo 2008

ISSN: $1697-7467$

\begin{abstract}
RESUMEN. El «slam» es una técnica poética en lengua oral que se recita en la Francia actual. Este artículo describe una serie de actividades realizadas en torno a un «slam», para trabajar las competencias comunicativas en clase de Français Langue Etrangère (FLE). Estas actividades, que tienen carácter interdisciplinar, ya que abordan contenidos de Historia y Geografía, presentan una Francia multicultural y ponen de manifiesto su diversidad lingüística. En el diseño de esta propuesta se ha tenido en cuenta el actual Marco Común Europeo de Referencia para las Lenguas.

Palabras claves: «slam», aprendizaje de la lengua francesa, Marco Común Europeo de Referencia para las Lenguas (MCERL), competencias generales, competencias comunicativas, Francia multicultural.
\end{abstract}

\section{A Didactic Proposal for Learning French through «slam»}

ABSTRACT. "Slam» is a poetic technique in oral language that is recited in present day France. This paper presents a group of activities carried out around a particular «slam» which are very useful for working on communicative skills on Français Langue Etrangère (FLE) lessons. These activities have an interdisciplinary nature since they deal with History and Geography knowledge . These activities provide an approach on a multicultural France and highlight linguistic diversity. This proposal follows requirements the Common European Framework of Reference for Languages.

Keywords: «slam», French language learning, Common European Framework of Reference for Languages (CEFRL), general competencies, communicative skills, multicultural France.

Versión actualizada y ampliada del artículo publicado en lengua francesa en Les langues modernes en abril de 2007.

\section{INTRODUCCIÓN}

Todas las propuestas de intervención en materia de enseñanza/aprendizaje convergen en las actividades. Mediante las mismas se aplican o ponen en uso todas las competencias adquiridas. Con ellas el profesor guía a sus alumnos para que trabajen los objetivos programados y son, también, el instrumento del que se vale para evaluar dichas adquisiciones.

Las actividades que aquí se presentan giran en torno a un documento auténtico que tiene la originalidad, además de servir para una clase de Français Langue Etrangère (FLE), de introducir contenidos de historia, geografía y cultura francesas. El género al que pertenece este documento es el «slam». 
El «slam» nace en los años ochenta en Chicago por iniciativa de un obrero de la construcción llamado Marc Smith que organizaba una serie de torneos poéticos en lengua oral. Los poemas se recitaban de forma peculiar imprimiendo «pulsos» en la entonación; de ahí viene su denominación ya que «to slam» en inglés significa golpear.

El «slam» es, entre otras cosas, una técnica poética en lengua oral. Hay tantas definiciones como «slameros» ${ }^{1}$ pero parece que existe un acuerdo en que debe respetar ciertas reglas: (i) aunque algunas veces puede ir acompañada de música de fondo, normalmente hip-hop o rap esta poesía debe ser dicha a capella; (ii) no debe exceder los tres minutos de duración; y (iii) debe realizarse en escenarios abiertos. El «slam» es un espectáculo que se presenta a modo de encuentro poético en el que cada cual se lanza para recitar libremente sus versos y compartirlos con los presentes. Los escenarios son muy dispares y a veces poco corrientes: lugares públicos como bares, cafés, librerías, metro, dando lugar a una reunión entre poetas y espectadores. Los temas que aborda son los de la vida cotidiana: "c'est de la poésie de proximité avec des mots de tous les jours » dice Grand Corps Malade (GCM), nombre artístico de uno de sus máximos exponentes en Francia, y el más mediático, como él mismo reconoce. El «slam» introduce una renovación en la poesía oral que a su vez hace resaltar el arte de la «performance» lingüística, lo que ha llevado a decir a GCM, en un «slam» de su último trabajo, depuis j'écris à l'oral ...

El entusiasmo suscitado por este género y la importancia que se le está otorgando ha dado lugar al nacimiento de talleres de «slam» ${ }^{2}$ en los colegios e institutos, y, más aún, algunos textos de este poeta han sido elegidos por el Ministerio de Educación para las pruebas de la selectividad francesa.

El hecho de que se presente este tipo de texto para trabajar la lengua francesa supone un acercamiento a un contexto, a veces, relegado por los profesores: la lengua oral, tal y como la hablan los jóvenes. De esta forma, se quiere presentar a los estudiantes una lengua más «familiar» y mostrarles una realidad a la que van a verse confrontados al realizar intercambios o estancias en Francia.

La utilización de la canción en las clases de FLE se ha entendido siempre como un soporte motivador que además permite trabajar las competencias comunicativas. Sin embargo, la elección de las canciones no es siempre fácil ya que no son siempre bien recibidas dado el público con el que se trabaja en las aulas: adolescentes, jóvenes adultos.

GCM, poeta de la actualidad, acompasa y cincela la letra de sus poemas con el «slam». Recuerda, por una parte la tradición de la canción francesa poética, hablada o recitada, interpretada por Barbara, Yves Montand, Jacques Brel, Serge Reggiani, Georges Brassens e incluso Jean Gabin ${ }^{3}$ y, por otra, Renaud con la utilización del «verlan» ${ }^{4}$ y la descripción de las ciudades del extrarradio parisino.

\footnotetext{
${ }^{1}$ Traducción literal del término original francés: slameurs.

${ }^{2}$ Fabien Marsaud (alias Grand Corps Malade) a pesar de su agenda sigue animando talleres de escritura en asociaciones culturales de su ciudad.

3 «Maintenant je sais» de Jean-Lou Dabadie, 1974. Album : Maître Corbeau et Juliette Renard.

${ }^{4}$ Es una lengua que se inventaron los jóvenes de los barrios del extrarradio parisino que recoge la pronunciación de «l'envers», palabra francesa que significa «al revés». El «verlan» escribe y pronuncia palabras al revés, un poco como los palíndromos.
} 
Desde la primera escucha, el «slam» Saint-Denis, escrito por GCM y publicado en su primer CD, llamó nuestra atención porque con él se puede introducir una clase de FLE a la vez que tratar temas de geografía, historia y cultura de Francia. Este «slam» es el que se va a utilizar como fuente para el diseño de las actividades que aquí se presentan y que se describen en los siguientes apartados de este artículo. El texto completo del «slam» se reproduce en el apartado 2 del artículo.

La ciudad de Saint-Denis, en la que GCM se crió y donde sigue residiendo, se presenta como un cruce de cultura y da la visión de esta Francia plural, mal aceptada y peor vista desde los acontecimientos de 2005, que GCM ilustra en otro «slam» diferente al antes mencionado, que aparece en su segundo $\mathrm{CD}$, con la siguiente sentencia:

Il faut voir à la télé comme on parle de là où je viens. Si jamais je connaissais pas, j’y emmènerai même pas mon chien.

En este mismo «slam», GCM vuelve a hablar de ella reafirmando sus raíces personales:

je viens de là où les mecs traînent en bande pour tromper l'ennui

je viens de là où en bas ça joue au foot la nuit

je viens de là où la langue est en permanente évolution verlan, rebeu, argot .......

je viens de là où on est un peu méfiant et trop souvent parano ......

je viens de là où des gens naissent, des gens s'aiment, des gens crèvent

$\mathrm{Tu}$ vois bien de là où je viens, c'est comme tout endroit sur terre

\section{El teXto : Saint-Denis ${ }^{5}$}

$J^{\prime}$ voudrais(a) faire un slam ${ }^{(f)}$ pour une grande dame que j'connais depuis tout petit

$J^{\prime}$ 'voudrais ${ }^{(a)}$ faire un slam ${ }^{(f)}$ pour celle qui voit ma vieille canne du lundi au samedi

$J^{\prime}$ 'voudrais ${ }^{(a)}$ faire un slam ${ }^{(f)}$ pour une vieille femme dans laquelle j'ai grandi $\operatorname{Denis}^{(d)}$

$J^{\prime}$ voudrais ${ }^{(a)}$ faire un slam pour cette banlieue nord de Paname ${ }^{(a)}$ qu'on appelle Saint-

Prends la ligne $D$ du $R E R^{(g)}$ et erre dans les rues sévères d'une ville pleine de caractère

Prends la ligne 13 du métro ${ }^{(g)}$ et va bouffer ${ }^{(a)}$ au $M_{c D o}^{(a)}$ ou dans les bistrots ${ }^{(a)}$ d'une $^{(2)}$ ville pleine de bonnes gos $^{(a)}$ et de gros clandos $^{(a)}$

Si t'aimes ${ }^{(a)}$ voyager, prends le tramway ${ }^{(g)}$ et va au marché( ${ }^{(g)}$. En une heure, tu traverseras Alger $^{(c)}$ et Tanger ${ }^{(c)}$.

Tu verras des You $^{(a)}$ gos $^{(e)}$ et des Ro ${ }^{(a)} \mathrm{ms}^{(e)}$, et puis j't'emmènerais(a) à Lisbonne ${ }^{(c)}$

Et à 2 pas de New-Deli ${ }^{(c)}$ et de Karashi ${ }^{(c)}$ (t'as ${ }^{(a)}$ vu j'ai révisé ma géographie), j't'emmènerai bouffer $^{(a)}$ du Mafé(h) à Bamako ${ }^{(c)}$ et à Yamoussoukro ${ }^{(c)}$

Et si tu préfères, on ira juste derrière manger une crêpe ${ }^{(h)}$ là où ça sent Quimper ${ }^{(c)} e t$ où ça a un petit air de Finistère ${ }^{(c)}$

Et puis en repassant par Tizi-Ouzou ${ }^{(c)}$, on finira aux Antilles ${ }^{(c)}$, là où il y a des grosses re noi $^{(b)}$ qui font "Pchit, toi aussi kaou ka fé la ma fille!" ()

\footnotetext{
${ }^{5}$ Se puede escuchar Saint-Denis en la web oficial de GCM: http://www.grandcorpsmalade. com/accueil.htm, y ver el vídeo en: http://ma-tvideo.france2.fr/video/iLyROoaftITX.html
} 
Au marché ${ }^{(g)}$ de Saint-Denis ${ }^{(c)}$, faut que ${ }^{(a)}$ tu sois sique-phy ${ }^{(b)}$. Si t'aimes pas être bousculé tu devras rester zen ${ }^{(t)}$

Mais sûr que tu prendras des accents plein les tympans et des odeurs plein le zen ${ }^{(b)}$

Après le marché on ira ché-mar ${ }^{(b)}$ rue de la République(d), le sanctuaire des magasins pas chers

La rue préférée des petites rebeus ${ }^{(b)}$ bien sapées ${ }^{(a)}$ aux petits talons et aux cheveux blonds peroxydés

Devant les magasins de zouk(t), je t'apprendrai la danse. Les après-midis de galère tu connaîtras l'errance. Si on va à la Poste(g) j't'enseignerai ${ }^{(a)}$ la patience ...

La rue de la République ${ }^{(d)}$ mène à la Basilique ${ }^{(d)}$ où sont enterrés tous les rois ${ }^{(d)}$ de France $^{(c)}$, tu dois le savoir! Après Géographie, petite leçon d'histoire

Derrière ce bâtiment monumental, j't'emmène ${ }^{(a)}$ au bout de la ruelle, dans un petit lieu plus convivial, bienvene au Café Culturel ${ }^{(g)}$

On y va pour discuter, pour boire, ou jouer aux dames. Certains vendredi soir, $y^{\prime} a^{(a)}$ même des soirées Slam ${ }^{(t)}$

Si tu veux bouffer ${ }^{(a)}$ pour 3 fois rien $^{(a)}$, j'connais ${ }^{(a)}$ bien tous les petits coins un peu poisseux ${ }^{(a)}$

On y retrouvera tous les vauriens ${ }^{(a)}$, toute la jet-set ${ }^{(t)}$ des aristocrasseux ${ }^{(a)}$

Le soir, y'a pas ${ }^{(a)}$ grand chose à faire, $y^{\prime}$ a pas ${ }^{(a)}$ grand chose d'ouvert Land $^{(b)}$

A part le cinéma ${ }^{(g)} d u$ Stade, où les mecs ${ }^{(a)}$ viennent en bande : bienvenue à Caillera-

Ceux qui sont là rêvent de dire un jour " je pèse! "(a) et connaissent mieux Kool Shen ${ }^{(f)}$ sous le nom de Bruno(f) Lopez $^{(e)}$

C'est pas une ville toute rose mais c'est une ville vivante. Il s'passe ${ }^{(a)}$ toujours quelqu'chose ${ }^{(a)}$, pour moi elle est kiffante ${ }^{(a)}$

$J^{\prime}{ }^{\prime}$ connais ${ }^{(a)}$ bien ses rouages, $j^{\prime}$ connais ${ }^{(a)}$ bien ses virages, $y^{\prime} a^{(a)}$ tout le temps du passage, $y^{\prime} a^{(a)}$ plein d'enfants pas sages, j'veux ${ }^{(a)}$ écrire une belle page, ville aux cent mille visages, St-Denis ${ }^{(c)}$-centre mon village

J'ai $93200^{(g)}$ raisons de te faire connaître cette agglomération. Et t'as ${ }^{(a)}$ autant de façons de découvrir toutes ses attractions.

A cette putain ${ }^{(a)}$ de cité j'suis $^{(a)}$ plus qu'attaché, même si j'ai envie de mettre des taquets $^{(a)}$ aux arracheurs de portables de la Place du Caquet

St-Denis ${ }^{(c)}$ ville sans égal, St-Denis ${ }^{(c)}$ ma capitale, St-Denis ${ }^{(c)}$ ville peu banale.. où à Carrefour $^{(g)}$ tu peux même acheter de la choucroute(h) Hallal (f)!

Ici on est fier d'être dyonisiens ${ }^{(c)}$, j'espère que j't'ai ${ }^{(a)}$ convaincu. Et si tu m'traites $^{(a)}$ de parisien $^{(c)}$, j't'enfonce $^{(a)}$ ma béquille dans l'... (Non!)

$J^{\prime}$ voudrais ${ }^{(a)}$ faire un slam ${ }^{(f)}$ pour une grande dame que j'connais ${ }^{(a)}$ depuis tout petit

$J^{\prime}$ voudrais(a) faire un slam ${ }^{(t)}$ pour celle qui voit ma vieille canne du lundi au samedi

$J^{\prime}$ 'voudrais ${ }^{(a)}$ faire un slam ${ }^{(f)}$ pour une vieille femme dans laquelle j'ai grandi Denis $^{(c)}$

$J^{\prime}$ voudrais ${ }^{(a)}$ faire un slam ${ }^{(f)}$ pour cette banlieue nord de Paname ${ }^{(a)}$ qu'on appelle Saint-

NOTA: Los 8 letras escritas como superíndices sirven para localizar todo el léxico necesario para la realización de las actividades de este «slam»:

1) lengua hablada y francés familiar ${ }^{(\mathrm{a})}$; $\operatorname{verlan}^{(\mathrm{b})}$

2) geografía $^{(\mathrm{c})}$; historia ${ }^{(\mathrm{d})}$

3) nacionalidades $^{(\mathrm{e})}$; cultura $^{(\mathrm{f})}$ : vida cotidiana ${ }^{(\mathrm{g})}$ y gastronomía $^{(\mathrm{h})}$ 


\section{El público}

Las actividades que aquí se presentan fueron propuestas a estudiantes de primer curso de la Diplomatura de Maestro de Lengua Extranjera (Francés) de la Universidad de Granada, en el marco de la asignatura Aspects socioculturels de la langue française.

Como punto de partida para su aplicación se utilizó la monarquía en la Historia de Francia. Se inició su desarrollo presentando de forma somera las dinastías más importantes del país. Llegados a la peculiar ceremonia de la consagración de los reyes de Francia, que tenía lugar casi siempre en Reims, se imponía una referencia a la ciudad de Saint-Denis, localidad en la que están enterrados la mayoría de los reyes franceses.

El desarrollo de las actividades ocupó cuatro sesiones de una hora, en ellas no se computa el tiempo empleado para la actividad de producción escrita que realizaron en horas no presenciales.

\section{LAS COMPETENCIAS TRABAJADAS}

A la hora de diseñar las actividades, se tuvo en cuenta las directrices expuestas en el Marco Común Europeo de Referencia para las Lenguas (2001), de ahora en adelante MCERL.

El «slam» Saint-Denis sirve para desarrollar ciertas competencias generales individuales $\mathrm{y}$, en particular, los saberes que pertenecen a la cultura general, el conocimiento del mundo y la diferencia intercultural.

Las actividades que se proponen ponen en funcionamiento las competencias comunicativas propuestas en el MCERL:

- lingüística (saberes y saber-hacer relativos al léxico, a la fonética, a la sintaxis).

- sociolingüística (conocer los parámetros socioculturales de la utilización de la lengua, en este «slam», los usos generacionales de la lengua, el empleo de la lengua por grupos sociales y la lengua francesa que proviene de una cultura diferente : la Antillana.

- pragmática (saber realizar las funciones comunicativas y reconocer los tipos de textos y géneros textuales, en este contexto: leer documentos históricos, diccionarios, diferentes textos en Internet y escribir un «slam»).

También tenemos en consideración el documento elaborado por el Consejo de Europa para Développer la dimension interculturelle dans l'enseignement des langues, que alienta a los profesores para que transmitan una «éducation à la compréhension interculturelle pour [promouvoir] une meilleure compréhension mutuelle et l'acceptation de la différence dans nos sociétés multiculturelles et multilingues» y que pretende « faire des apprenants des locuteurs ou médiateurs interculturels capables de [...] s'engager dans un contexte d'identités multiples » y en el que se recomienda «... d'utiliser du matériel de caractère authentique».

Este documento auténtico, Saint-Denis, permite al alumno:

- desarrollar su competencia intercultural

- conocer otros grupos sociales, sus costumbres y sus prácticas, 
- reconocer diferentes registros de la lengua francesa,

- aumentar su vocabulario,

- aprender a pronunciar con el flujo adecuado marcado por la rima,

- fijar estructuras de la lengua oral.

\section{Propuestas metodológicas}

La clasificación temática de las actividades que se muestra en este apartado se debe a razones formales ya que algunos contenidos podrían perfectamente ser transferibles a otros apartados. Las indicaciones y consignas para realizarlas se han dado en francés. Y excepto las lecturas silenciosas y las lecturas en voz alta, obviamente, se les pidió que las realizaran en cooperación.

\subsection{Desarrollar las competencias comunicativas:}

\subsubsection{Lingüistica:}

- Fonética

- Después de dos escuchas seguidas, repartir y presentar la letra del «slam». Se pide a los estudiantes que realicen una lectura silenciosa y que subrayen las palabras que transcriben la lengua hablada: j'voudrais, j'connais, t'aimes, t'as, 2 pas, faut que, gos $^{6}$, etc. .

- Al final, después de realizar las distintas actividades de esta propuesta didáctica, pero antes de la actividad de creación escrita, y después de varias escuchas, el alumno leerá en voz alta para familiarizarse con el texto.

- Semántica

- Mismo procedimiento que para el descubrimiento del francés familiar de los jóvenes: realizan una nueva lectura silenciosa para localizar las palabras clandos, Yougos, Roms, bouffer, sapées, mecs, kiffante, etc., pero en este caso pueden necesitar la ayuda del profesor. A continuación, se dan explicaciones para ciertas palabras como: Yougos, Roms, je pèse, kiffante, taquets. Finalmente, se solicita que sustituyan todos esos vocablos por los correspondientes al registro de lengua estándar.

- Morfosintaxis

- A continuación, han de proceder a escribir las palabras y frases que representan la lengua oral según las reglas de morfosintaxis: j'voudrais/je voudrais, t'aimes/tu aimes, faut quel il faut que,... il n'y a pas, il y a, etc.

\subsubsection{Sociolingüistica}

- Tras la explicación ofrecida sobre el origen del «verlan», tienen que encontrar en el texto las tres palabras más evidentes que representan este peculiar lenguaje: re-noi, sique-phy, ché-mar.

\footnotetext{
${ }^{6}$ Escrito «gos» por el autor. Pensamos que el significado es el de «gosse».
} 
Las otras tres más difíciles de detectar se las indicamos directamente: zen [nez], rebeus [beurs], Caillera-Land [racaille-land].

- Con un pequeño comentario, se explica el origen y significado de la palabra «Paname»: proviene del griego «panamis» y significa capital. Se les pide que recuerden otras dos denominaciones para la capital de Francia que aprendieron en el tema de Geografía: Lutèce y Paris.

- Como actividad de producción escrita, se invita a los estudiantes a que inventen algunas palabras en «verlan».

\subsubsection{Pragmática}

Esta competencia se trabaja a través de varias tareas realizadas también en cooperación ${ }^{7}$ como: leer documentos históricos sobre la ceremonia de consagración de los reyes de Francia, diccionarios, diferentes textos en Internet y escribir un «slam».

\subsection{Enriquecer los saberes de las otras disciplinas :}

\subsubsection{Geografia}

- 93200: (93 es el departamento de Seine-St-Denis). Se les pide que oralmente recuerden la división territorial de Francia que han estudiado en temas anteriores: région, département, commune, les DOM-TOM

- Una nueva lectura del «slam» les sirve para localizar las palabras que se refieren a lugares geográficos: ciudades, países, islas (St-Denis, Alger, Quimper, Finistère, France, Antilles, etc.) así como los gentilicios (dionysiens, parisiens). Se les propone que den el que corresponde a las Antilles ya que no aparece en el texto del «slam».

- En otro momento, han de localizar los restantes lugares utilizando Internet tanto en un mapa mundi, como en un mapa de Francia. Se emplea el mismo procedimiento para la ciudad de Saint-Denis donde manejarán un plano virtual de la ciudad.

- Finalmente, indicar a los estudiantes que el Finisterre español tiene también un correspondiente francés y recurriendo a Internet situarán a ambos geográficamente.

\subsubsection{Historia}

Los estudiantes tienen que averiguar los siguientes datos, para ello se prevé una sesión en el aula de informática:

- el personaje de Saint Denis,

\footnotetext{
${ }^{7}$ «Pour qu'on puisse dire qu'une activité est une tâche, il faut qu'elle soit réalisée en coopération [...] Elle peut donc impliquer l'utilisation [...] de toutes ces compétences à la fois. [...] met en place des processus cognitifs, $[\ldots]$ : les apprenants doivent atteindre ensemble un objectif, $[\ldots]$ devront se mettre d'accord sur la façon d'y arriver, choisir l'information importante à leurs yeux, valoriser différents avis et choix de langues, etc. Ces processus cognitifs sont beaucoup plus complexes que ceux qui sont employés dans une activité où il n'y a qu'à répéter un modèle linguistique prédéfini. Elle aboutit à un produit clairement communicatif, objectif final d'une tâche. (une fiche avec les renseignements personnels d'un camarade de classe, un poème, une liste de conseils....)». Emilia Conejo López-Lago (2007).
} 
- la ceremonia de la consagración de los reyes de Francia,

- las fechas y las razones de la construcción de la Basílica,

- la fecha del nacimiento de la República francesa - puesto que GCM habla de la calle de la République en Saint-Denis-,

- la reciente historia del Stade de France, lugar de éxitos deportivos (les señalaremos y explicaremos la expresión «black-blanc-beur » nacida en el mundial de fútbol de 1998).

\subsection{Desarrollar el saber-hacer:} fin de:

Para llevar a cabo lo siguiente se continua trabajando en el aula de informática con el

- visitar la página web http.//maps.google.fr y localizar en Saint-Denis la calle de la République, el itinerario para ir a la Basílica, para llegar a la Place du Caquet así como al Café Culturel.

- realizar una visita virtual de la Basílica de Saint-Denis: http://www.tourisme93.com/ basilique/.

- realizar otra visita virtual, esta vez, al Café Culturel, lugar en el que GCM «slamea» en Saint-Denis el segundo viernes de cada mes. Puede visitarse en: http:// www.cafeculturel.org/.

\subsection{Desarrollar la competencia intercultural:}

Se vuelve al texto para realizar otras búsquedas, documentarse y adquirir conocimientos sobre:

\subsubsection{Las diferentes nacionalidades}

- Yougos[laves], Roms (de «Roumains» o «romanichels»), Beurs (personas de origen magrebí), Lopez (apellido de origen español).

\subsubsection{Los elementos culturales}

- Magasin de zouk: el zouk es un género musical, nacido en 1980, interpretado en la Martinica y la Guadalupe. Esta palabra tendría su origen en mazouk, la mazurca criolla.

- Negras antillanas con su hablar (mezcla de Francés y Criollo): «Pchit, toi aussi kaou ka fé la ma fille ! » cuya traducción es: Mais enfin, que fais-tu ici ma fille!

- Zen : filosofía japonesa.

- La jet-set: apócope del inglés "jet society".

- Slam: herramienta de democratización y arte de técnica poética. El «slam» es la unión entre la escritura y la técnica que anima a los poetas a hacer hincapié en lo que dicen y en cómo lo dicen. «Slamear» ${ }^{8}$ es «golpear» las palabras en una fusión de poesía y rap acústico.

\footnotetext{
${ }^{8}$ Este verbo es nuestro. Lo hemos formado siguiendo la regla de rapero/rapear.
} 
- Hallal: término utilizado cuando se sacrifica un animal según la tradición islámica.

\subsubsection{La vida cotidiana}

Explicación y localización de ciertos lugares:

- RER : explicar las siglas Réseau Express Régional y observar sobre un plano de París el itinerario recorrido por la línea D del RER.

- Línea 13 del metro : mirar sobre un plano del metro de París el itinerario que recorre dicha línea.

- El mercado: en Francia la mayoría de los mercados son al aire libre .

- El Café (Culturel): lugar de reunión de los jóvenes franceses.

- El cine (pedirles que den el título de varias películas francesas).

- Carrefour (pedirles que den otros nombres de grandes superficies francesas que se hallen en España: por ejemplo Auchan, Intermarché, Leroy Merlin, Promod, etc.).

\subsubsection{El vocabulario de la gastronomía:}

- Mafé: ragut africano de carne o de pescado con salsa de cacahuetes.

- Crêpe: delgada tortita de origen bretona, hecha de una masa líquida que hay que cocinar en una sartén. (Podemos recordarles la receta...).

- Choucroute: plato de Alsacia compuesto por col macerada en vino, panceta, salchicha, carne de cerdo ahumado, etc.

- Hallal: se dice también de las carnicerías que venden carne de animal sacrificado según la tradición islámica.

\subsubsection{Los sonidos}

Les proponemos que reconozcan los diferentes sonidos de fondo:

- Campanario/carillón de la Basílica de Saint-Denis,

- RER/metro: parada del metro; puertas que se abren y cierran

- En la calle: pasos con tacones, gritos, coches, viandantes,

- Las fichas del juego de damas en el Café Culturel.

\section{PARA IR MÁS LeJOS}

\subsection{Actividades de producción escrita}

El nivel general de lengua que poseen los alumnos es un nivel A1 y A2, aunque los hay también nativos, y no dominan la lengua francesa al punto de convertirse espontáneamente en poetas. Consciente de la dificultad que supone constreñirlos a escribir un texto poético, les sugerimos una serie de temas (le train, la rue, les voyages ...) de manera a que puedan elaborar un glosario previo que les sirva de base léxica suficiente para escribir un «slam» ${ }^{\text {, }}$ para ello nos apoyamos en una actividad que presenta le libro Activités pour le Cadre européen

\footnotetext{
${ }^{9}$ Algunos aparecen reproducidos en el anexo.
} 
commun de référence pour les langues. Dicha actividad, que les permite ejercitar su creatividad y descubrir la utilización poética de la lengua, resulta más fácil si la realizan en cooperación.

\subsection{Actividades de reproducción oral}

Se trata de recitar el «slam» que cada uno ha compuesto y de practicar por segunda vez la lectura en voz alta.

\subsection{Actividades para ampliar y profundizar la competencia intercultural}

En otro momento, trabajar el texto de otro «slam» de GCM : Je viens de là, haciendo hincapié en estas aseveraciones : " ... à chacun son territoire, à chacun sa France», "je viens de là où la France est un pays cosmopolite», "... je viens de la banlieue ».

\section{Conclusión}

El «slam» nuevo género poético oral, acompañado a veces con música de fondo, más audible que las canciones convencionales y más cercano a los jóvenes, resulta ser un buen instrumento para la escucha, la recitación, el trabajo del flujo, la lectura en voz alta y la creación en lengua francesa.

Está teniendo un gran éxito en las clases de FLE porque toca muy de cerca el corazón de los jóvenes por su lenguaje, sus temas y su estilo.

Al invitar a los estudiantes a conocer la ciudad de Saint-Denis en la que se encuentra la Basílica de los reyes de Francia a través del «slam», algunos pensaron que iban a ver un reportaje en DVD, otros que iban a descubrir una página web pero ninguno pensó en un género poético. Esto se debe a que no es conocido en España. La presentación de la cultura plural de Francia mediante este documento resulta ser atractiva y motivadora, y les sirve para conocer este género así como, en otro momento, apreciar otro tipo de música francesa.

Valoran la originalidad de la propuesta y la del texto del que aprecian las rimas, la cadencia de las palabras, la ausencia de música y en un segundo tiempo el vocabulario.

El lenguaje poético, que en un primer momento puede plantearles algunas dificultades a la hora de comprender el texto, se revela de gran ayuda para la lectura en voz alta o recitación gracias a la repetición de sonidos, y los anima para iniciarse en la producción escrita utilizando la lengua francesa de manera más creativa. Por otra parte, los juegos de palabras y el «verlan» dificultan la comprensión inicial del «slam».

Se puede concluir afirmando que mediante esta propuesta de actividades variadas los estudiantes ponen en funcionamiento sus competencias generales, desarrollan las comunicativas, la intercultural, así como aprenden diferentes contenidos que forman parte de otras disciplinas.

En lo que se refiere a las competencias generales, saber hacer, la localización de páginas web no presenta, para la mayoría, ninguna dificultad ya que poseen dicha habilidad. Los pocos que aún no lo habían hecho nunca fueron asesorados por los compañeros.

Este «slam» es además un documento interactivo en el que contenidos de los temas interdisciplinares, que forman parte del programa de la asignatura, se introducen de un modo más activo, y es capaz de suscitar una gran participación y entusiasmo. España se acerca más 
a Francia y viceversa con una cultura común: la celta, a través de un lugar de idéntica denominación: Finisterre/Finistère; recuerdan la diferencia entre los DOM y los TOM presentada en temas anteriores; recorren lugares del mapamundi cuya población está presente en la ciudad de Saint-Denis. En cuanto a la Historia de Francia, acceden a textos que explican el hecho del origen divino de la ceremonia de consagración de los reyes franceses, visitan páginas web para ver el lugar en el que están enterrados muchos de ellos a semejanza del Escorial en España, etc..

El desarrollo y el trabajo de las competencias comunicativas ha mejorado:

- aspectos fonéticos, desarrollando la habilidad de reconocer, previamente mediante la escritura, la lengua oral, y practicando, en varias ocasiones, la lectura en voz alta, lo que da lugar a una mejor comprensión del texto que deriva en la producción del flujo adecuado para la lectura en voz alta.

- aspectos semánticos, aprendiendo nuevas palabras, que son las que utilizan con mucha frecuencia los jóvenes franceses. Palabras que, en un segundo momento comprueban que ya conocen en francés estándar como: sapées/habillées; bouffer/manger; mecs/ hommes/garçons, etc..

- la lengua, realizando un trabajo de reescritura y utilizando para ello las competencias conceptuales y procedimentales relativas al léxico, a la sintaxis y otras dimensiones del sistema de la lengua francesa.

- Ha aumentado su competencia sociolingüística, al aprender ciertos vocablos en «verlan» y al construir algunas palabras en francés de esta guisa, como se puede apreciar en algunos de los «slams» que han escrito (ver anexo). Ahora bien, el desarrollo de la competencia pragmática, trabajada en varios momentos, fue la más difícil por dos razones:

a) los textos escritos en Francés que tienen que leer para extraer información presentan un nivel de lengua elevado que intentan salvar utilizando diccionarios online $\mathrm{y}$, en los casos más difíciles, preguntando a la profesora.

b) el ejercicio de producción y utilización de la lengua de forma creativa y poética es todo un reto que les proporciona enriquecimiento desde el punto de vista lingüístico y creativo, conscientes de la dificultad que entraña este tipo de ejercicio aun en lengua materna (ver anexo).

Por otra parte, amplían su competencia intercultural porque aprenden que hablar de Francia no es solamente hablar de lo genuinamente francés sino de otras nacionalidades y, por lo tanto, de otras costumbres que la conforman. Todo ello sirve para mejorar la comprensión del panorama histórico-social, así como para aceptar las diferencias y valorar el enriquecimiento que esto supone. De este modo, estos estudiantes serán más permeables cuando realicen sus prácticas de clase en centros escolares tanto en España como en Francia.

En general, el desarrollo de las habilidades y competencias en cualquier proceso de aprendizaje necesita del trabajo continuado y, a veces, repetitivo. Si además este proceso lo trasladamos a una lengua extranjera entendemos que las dificultades se acrecientan debido a que el canal de transmisión, la lengua, no se maneja con soltura. Por este motivo, y para que la utilización creativa de la lengua francesa no se quedara en una mera actividad puntual seguimos trabajando y animando sesiones para la producción de textos poéticos paralelamente 
al programa de las distintas asignaturas de lengua francesa. Iniciamos esta experiencia en el curso 2006/2007 y continuamos en el siguiente. De este modo, esta tarea continuada ha dado lugar a varias ediciones de producción de «slam» en la que los ganadores, siempre un chico y una chica, por edición, reciben un premio y todos los participantes exponen sus creaciones en las paredes de la clase.

Este gusto por el «slam» traspasó el contexto del aula, asistiendo a varias «soirées slam» ${ }^{10}$ organizadas por la «Maison de France», en Granada, a partir de noviembre de 2007, de esta manera pudieron comprobar en vivo cómo se desarrollan. En ellas, los más atrevidos subieron al escenario a recitar sus textos.

\section{ReFEREncias}

Bleser, N. (2006). «Un Grand Corps Malade qui soigne si bien ses mots» dans Logosphère, vol.2, 1-20, Revue d'Etudes Linguistiques et Littéraires. Université de Grenade.

Cadre européen commun de référence pour les langues, 2001, Paris: Didier.

Développer la dimension interculturelle dans l'enseignement des langues. Conseil de l'Europe, Strasbourg, 2002.

Cornaire, C. (1999). Le point sur la lecture, Coll. Didactique des langues étrangères. Paris: Clé International.

Fiasse, I. (2000). «Approche des expressions idiomatiques en F.L.E.» dans Le langage et l'homme, vol XXXV no 1 Ed. Peeters.

Conejo López-Lago, E. (2007). Descargado el 7 de enero de 2008. http://www.francparler.org/ dossiers/pj/conejo.pdf

Goullier, F. (2006). Les outils du Conseil de l'Europe en classe de langue. Paris: Didier.

Grand Corps Malade, «Saint-Denis»: chanson $n^{\circ} 2$ du Cd Midi 20, 2006, Universal Music France S.A.S.

Grand Corps Malade, «Je viens de là»: chanson $n^{\circ} 2$ du Cd Enfant de la ville, 2008, Universal Music France.

http://www.grandcorpsmalade.com/accueil.htm: Site officiel de Grand Corps Malade.

http://www.tourisme93.com/basilique/: Site de l'Office du Tourisme de Seine-Saint-Denis.

http://www.ffdsp.com/: Site officiel de la fédération française de Slam.

http.//maps.google.fr: Site de maps.google.fr

Parizet, M-L., Grandet, E., Corsain, M. (2005). Activités pour le Cadre européen commun de référence pour les langues. Niveau A2. Paris: Clé international.

Urbano, B. (2000). «Lectura de un texto musical. Explotación didáctica de un anti-modelo textual» en El reto de la lectura en el siglo XXI, 789-802. Granada: GEU.

Urbano, B. (2002). «Léa de Louise Attaque» dans Cahiers Pédagogiques, 395,67-68, Paris: C.R.A.P.

Urbano, B. (2007). «Ça va chémar en classe de langues. Le slam de Grand Corps Malade: multiculturalité, lectures à haute voix et interdisciplinarité» dans Les langues Modernes, 2/ 2007, 84-89, Paris: APLV.

Vielmas, M.(1990). A haute voix, Coll. Techniques de classe. Paris: Clé international.

\footnotetext{
${ }^{10}$ La primera «soirée» fue inaugurada por Frédéric Nevchehirlian (http://www.myspace.com/nevchehirlian) poeta-slamero que además es vocalista del grupo francés Vibrion.
} 


\section{Anexo}

\section{Primera edición}

\section{Poétique du train}

Il y a ceux qui ne trouvent pas leur place

Celles qui font de grands signes à ceux qui restent

sur le quai

Ceux qui écoutent de la musique très fortement

Celles qui parlent au téléphone pendant tout

$$
\text { le voyage }
$$

Ceux qui regardent tristement par la fenêtre

Ceux qui lisent les magazines, les journaux, les livres

Celles qui écrivent dans leur journal ou quelques

lettres aux amis

Celles qui causent avec chacun et chacune

Et aussi ceux qui dorment pendant tout

leur voyage.

Stefanie Peuser

\section{Le train bleu}

Voilà ceux qui se promènent en regardant le ciel ...

Un ciel fermé et ouvert à la fois.

Voilà ceux qui parlent tous seuls sur les quais

Avec leur solitude.

Le train bleu va partir et par contre ...

Les voyageurs ne regardent pas les nuages.

Sonia Hurtado Peña

\section{Segunda edición}

\section{Slam des rues perdues}

L'émotion de la vie je la trouve dans la rue, J'étais abattue et sans rire dans le reaubu ${ }^{11}$ :

Plus d'espace, noir absolu

Et le chercheur de rêves dans l'avenue

Je suis dans la rue avec son air pur te ses émotions, mon inspiration

Je m'assieds sur un banc plein de bonbons, quelle situation !!!

C'est bizarre mais tout de suite viennent les enfants,

Instinct de protection

Je comprends et je les leur donne.

\footnotetext{
${ }^{11}$ Bureau
} 
$\mathrm{Tu}$ veux une explication

Je suis le berger de tous ces moutons

Je commence à faire le slam,

Slam des rues perdues,

Rues que je n'ai jamais vues

Admire leurs détails, leurs différences:

Rues, rues;

Pleines de gens qui les oublient:

rue pure

Et cherche son âme, tu seras prissur ${ }^{12}$

Laura Marruecos Mansilla

\section{Mon premier slam}

C'est mon vocabulaire qui n'a pas beaucoup de mots

Alors ce que je veux faire

C'est simplement un slam

D'autres choses, n'importe quoi!

Ja! Comme le roi,

Grand Corps Malade!

Pour lui un seul mot dans mon cerveau:

Sans REDDITION, sans exceptions:

C'est quoi ton problème mec?

$\mathrm{Tu}$ veux queque chose mieux?

Ah non! Tu devras prendre ton cahier ....

Compose avec moi ! C'est vachement sympa!

Un, deux, trois !!! ..ici, mon premier slam!!!!

David Zafra Cámara

Le SLAM et les arts de la rue,

Sentir que tu es pas perdu...

Dans la vie il y a des hauts et des bas,

Parfois tu te demandes qu'est-ce que tu fous là

Mais il y a toujours quelque chose près de toi

Pour te faire penser, «arrête, décone pas!»

Combattre les fantômes qui essaient de t'enfoncer.

Et plus tard tu te dis que tu as bien fait,

De ne pas trépasser

Nathalie Deline Mullis

\section{Je rêve ma rue parfaite}

Je ne sais pas faire un slam

$\mathrm{C}^{\prime}$ est pour ça que personne ne peut dire qu'il est lam (mal)

Dans ma rue nous sommes tous frères et sœurs, nous sommes heureux, même si on n'est pas des slameurs.

${ }^{12}$ Surpris 
Les blancs, les noirs et tous chantons ces chansons, nous cherchons une rue où toutes les personnes soient très bonnes.

À cette époque- la, j'ai cherché l'amour parfait, je ne l'ai pas trouvé et pour cela je m'en suis allé, tout imparfait.

Ce rêve s'est cassé et maintenant je reviens pour si tout va bien, je regarde au ciel et il continue d'être cruel, cela est réel, mais je continue de rêver de ma rue idéale, elle est égale, je l'obtiendrai parce que je lutterai.

Je ne sais pas faire un slam,

Mais c'est la raison de mon illusion, je rêve ma rue parfaite.

Jorge Alberto Peláez Herrera

\section{La nuit}

Aujourd'hui je suis perdu

Dans le slam et dans la rue

La rue comme une forêt noire

Avec des lianes et des singes.

Aujourd'hui je suis perdu

Dans le slam et dans la rue

La rue comme une caverne noire

Avec des voleurs et des clochards.

Aujourd'hui je suis perdu

Dans le slam et dans la rue

La rue comme un marécage

Avec des monstres et de la boue.

Bienvenue à la nuit et à la rue!

Mathias Vinuesa Vigilia

\section{Mon slam}

J' habite avec un chauve et un musclé,

Le chauve il se fait insulté ${ }^{13}$,

Pas le musclé, puisqu' il peut me frappé.

Chez moi personne fait la vaisselle,

Il paraît qu'un typhon est passé dans la cuisine,

Il est vu que chez nous il n'y a pas une mademoiselle.

Dans ma maison il y a une chaise défaite,

Quand quelqu'un est assis,

Il prends le risque de se cassé la tête.

Sergio López Marín

\footnotetext{
${ }^{13}$ Las incorrecciones las dejamos tal y como los estudiantes las escribieron.
} 


\section{Tercera edición}

Je pars en voyage pour me rencontrer.

Sur le sommet d'une montagne solitaire,

Devant mes yeux ouverts,

Un cadre jamais peint, jamais vécu,

Mais fait réalité, devant mon corps fatigué.

\section{C'est un froid hiver}

Heureusement, c'est ce que je préfère

Je suis émerveillée par la vallée,

Ma nouvelle maison où je vivrai

Pendant les prochains mois, tranquille, reposée

Je me lève tôt tous les matins,

Et je me lave avec de l'eau fraîche

Les heures passent, je suis appuyée à la fenêtre,

Je regarde les formes des nuages:

Ils ressemblent à la neige blanche.

Parfois les voyages coûtent cher

Mais coûte que coûte

On a besoin de les faire

Pour retourner chez quelqu'un

Avec un sourire sur le visage

Lucía Quijano

\section{Un voyage comme la nuit}

un jour j'aimerai faire un voyage avec les yeux bandés

n'importe ni le chemin ni le lieu où on va

puisque je sais que tu es là

je me sens pas nerveux

puisqu'une seconde avec toi

ça me rend heureux

je ne sais pas si on va loin ou tout près

je ne sais pas si on vole ou on va à pied

n'importe si le chemin est long

je sens tes doigts entre les miens

je sens que tu es là

tout près de moi

Mathias Vinuesa Vigilia 